\title{
MHD Fluctuating Flow of Non-Newtonian Fluid through a Porous Medium Bounded by an Infinite Porous Plate
}

\author{
Dhiman Bose, Uma Basu \\ Department of Applied Mathematics, University of Calcutta, Kolkata, India \\ Email: dhimanbose09@gmail.com, umabasu49@gmail.com
}

Received 13 January 2015; accepted 8 November 2015; published 11 November 2015

Copyright (C) 2015 by authors and Scientific Research Publishing Inc.

This work is licensed under the Creative Commons Attribution International License (CC BY). http://creativecommons.org/licenses/by/4.0/

c) (i) Open Access

\begin{abstract}
In present paper, an investigation has been made on the fluctuating flow of a non-Newtonian second grade fluid through a porous medium over a semi-infinite porous plate in presence of a transverse magnetic field $B_{0}$. The governing equations have been solved analytically and the expressions for the velocity and stress fields are obtained. The free stream velocity $U(t)$ fluctuates in time about a non-zero constant mean. The effects of the permeability parameter $K$ and magnetic field parameter $M$ on velocity field have been analyzed quantitatively with the help of figures. It is noticed that the velocity field asymptotically approaches free stream velocity as it goes far away from the plate.
\end{abstract}

\section{Keywords}

Fluctuating Flow, Second Grade Fluid, Porous Medium, Transverse Magnetic Field, Free Stream Velocity

\section{Introduction}

The fluctuating flows of viscous incompressible fluid through porous medium over an infinite porous plate have been investigated rigorously by many researchers because of its wide application in different fields. The phenomenon of flows through porous medium has been a subject of interest of many researchers because of its wide range of application in different fields such as petroleum engineering, chemical engineering etc. In petroleum engineering, it is dealt with the movement of natural gas and oil through reservoirs. Further, the study on underground water resources, seepage of water in river bed is also related to the flow through porous medium.

Soundalgekar and Puri [1] investigated on fluctuating flow of an elastic-viscous fluid past an infinite plate 
with variable suction. Shen, Tan, Zhao and Masuoka [2] studied the Rayleigh-Stokes problem for a heated generalized second grade fluid with fractional derivative model. Varshney [3] studied fluctuating flow of viscous fluid through a porous medium bounded by a porous plate. In his work, he has used Lighthill's method in finding the solution of the field equation and shows that the results obtained reduce those of Messiha [5]. Lighthill [4] initiated the work on fluctuating flows. He studied the response of laminar skin friction and heat transfer to fluctuations in the stream velocity. An important class of two dimensional time dependent flow problems dealing with the response of the boundary layer to unsteady fluctuations about a mean value has been studied by him. Messiha [5] investigated laminar boundary layer in oscillating flow along an infinite flat plate with variable suction. Stuart [6] studied a solution of the Navier-Stokes and energy equations illustrating the response of skin friction and temperature of an infinite plate thermometer to fluctuations in the stream velocity. Gholizadeh [7] discussed MHD oscillatory flow past a vertical porous plate through porous medium in the presence of thermal and mass diffusion with constant heat source. Moniem and Hassanin [8] investigated solution of MHD flow past a vertical porous plate through a porous medium under oscillatory suction. Venkateswarlu, Reddy and Lakshmi [9] studied unsteady MHD flow of a viscous fluid past a vertical porous plate under oscillatory suction velocity. Soundalgekar and Takhar [10] discussed MHD oscillatory flow past a semi infinite plate.

In the present study, we have investigated the fluctuating flow of non-Newtonian fluid in a semi-infinite region through a porous medium bounded by a porous plate in presence of transverse magnetic field. The nonNewtonian fluid considered here is of second grade type and fluctuating flow related to such type of fluid is different from all those works that have been done earlier. The analytical solutions for the velocity and stress fields have been obtained by Lighthill's method. The effects of permeability parameter $K$, Hartmann number $M$, frequency of fluctuation $\omega$ on the velocity field and skin friction have been illustrated graphically.

\section{Mathematical Analysis of the Problem}

Let us consider the flow of a second grade fluid of density $\rho$ and viscosity $\mu$ through a porous medium of permeability $K$ occupying a semi infinite region of the space bounded by a porous plate in presence of a transverse magnetic field $B_{0}$. At time $t \leq 0$ the fluid and the plate are at rest and at $t>0$ the fluid begins to move due to a pressure gradient $\frac{\mathrm{d} p}{\mathrm{~d} x}$. Let $u$ and $v$ are the components of velocity in $x$ and $y$ directions taken along and perpendicular to the porous plate. It is assumed that $U(t)$ be the free stream velocity parallel to the plate which fluctuates in magnitude about a mean free stream velocity $U_{0}$ in the direction of $x$-axis. Since the plate be situated in the $x z$-plane and the plate is of infinite dimension in $x$ and $z$ direction, all the quantities are only functions of $y$ and $t$.

The equation of motion for the incompressible viscous generalized second grade fluid can be written as

$$
\begin{gathered}
\frac{\partial u}{\partial t}+\frac{v}{\rho} \frac{\partial u}{\partial y}=-\frac{1}{\rho} \frac{\partial p}{\partial x}+\left(v+\alpha \frac{\partial}{\partial t}\right) \frac{\partial^{2} u}{\partial y^{2}}-\frac{\mu u}{\rho K}-\frac{\sigma}{\rho} B_{0}^{2} u \\
\frac{\partial v}{\partial t}=-\frac{1}{\rho} \frac{\partial p}{\partial y}-\frac{\mu v}{\rho K}
\end{gathered}
$$

The equation of continuity

$$
\frac{\partial v}{\partial y}=0
$$

$v=\frac{\mu}{\rho}$ is the kinematic viscosity, $\alpha=\frac{\alpha_{1}}{\rho}, \alpha_{1}$ is normal stress moduli, $\rho$ is the fluid density, $\mu$ is the viscosity of the fluid, $K$ is the permeability of the porous medium, $\sigma$ is the electrical conductivity.

For free stream

$$
\frac{d U(t)}{d t}=-\frac{1}{\rho} \frac{\partial p}{\partial x}-\frac{\mu U}{\rho K}-\frac{\sigma}{\rho} B_{0}^{2} U
$$

Let the fluctuating stream and suction velocities of the form 


$$
\begin{gathered}
U(t)=U_{0}\left(1+\varepsilon \mathrm{e}^{i \omega t}\right) \\
v=-v_{0}\left(1+A \varepsilon \mathrm{e}^{i \omega t}\right)
\end{gathered}
$$

where $U_{0}$ and $v_{0}$ be the mean free stream and mean suction velocity respectively, $\varepsilon \ll 1$ and $A$ is such that $A \varepsilon \leq 1 ; \omega$ is the frequency of fluctuating stream.

The boundary conditions can be written as:

No slip boundary condition $u=0$ at $y=0$.

And

$$
u=U \text { as } y \rightarrow \infty
$$

Eliminating $\frac{1}{\rho} \frac{\partial p}{\partial x}$ between Equation (1) and Equation (4) we obtain

$$
\frac{\partial}{\partial t}(u-U)+v \frac{\partial u}{\partial y}=\left(v+\alpha \frac{\partial}{\partial t}\right) \frac{\partial^{2} u}{\partial y^{2}}-\frac{\mu}{\rho K}(u-U)-\frac{\sigma}{\rho} B_{0}^{2}(u-U)
$$

\section{Method of Solution}

Let us introduce the following non-dimensional quantities

$$
y^{\prime}=\frac{U_{0} y}{v}, t^{\prime}=\frac{U_{0}^{2} t}{v}, U^{\prime}=\frac{U}{U_{0}}, u^{\prime}=\frac{u}{U_{0}}, v^{\prime}=\frac{v}{v_{0}}, K^{\prime}=\frac{U_{0}^{2}}{v^{2}} K, M^{2}=\frac{\sigma}{\rho} \frac{v}{U_{0}^{2}} B_{0}^{2}
$$

where $M=\sqrt{\frac{B_{0}}{U_{0}}} \frac{\sigma v}{\rho}$ is the Hartmann number, $K^{\prime}$ is the non-dimensional permeability parameter.

The Equation (8) in terms of non-dimensional variables reduces to (Dropping ' sign for convenience)

$$
\frac{\partial}{\partial t}(u-U)+v \frac{\partial u}{\partial y}=\left(1+m \frac{\partial}{\partial t}\right) \frac{\partial^{2} u}{\partial y^{2}}-\frac{1}{K}(u-U)-M^{2}(u-U)
$$

where $m=\frac{\alpha}{v^{2}} U_{0}^{2}$.

In non-dimensional variables Equation (5) and Equation (6) becomes

$$
\begin{aligned}
& U=1+\varepsilon \mathrm{e}^{i \omega t} \\
& v=-\left(1+A \varepsilon \mathrm{e}^{i \omega t}\right)
\end{aligned}
$$

The boundary conditions (7) in non-dimensional variables can be written as

$$
u=0 \text { at } y=0 \text { and } u=U \text { as } y \rightarrow \infty
$$

Let the velocity in the neighborhood of the plate be

$$
u=\left(\psi_{0}(y)+\varepsilon \mathrm{e}^{\mathrm{i} \omega t} \psi_{1}(y)\right)
$$

Substituting for $u$ and $U$ from Equation (13) and Equation (11) respectively in Equation (10) and separating harmonic and non-harmonic terms and neglecting the squares of $\varepsilon$ we obtain

$$
\begin{gathered}
\frac{\mathrm{d}^{2} \psi_{0}}{\mathrm{~d} y^{2}}+\frac{\mathrm{d} \psi_{0}}{\mathrm{~d} y}-\left(\frac{1}{K}+M^{2}\right) \psi_{0}=-\left(\frac{1}{K}+M^{2}\right) \\
\frac{\mathrm{d}^{2} \psi_{1}}{\mathrm{~d} y^{2}}+\frac{1}{1+m i \omega} \frac{\mathrm{d} \psi_{1}}{\mathrm{~d} y}-\left(\frac{\frac{1}{K}+M^{2}+i \omega}{1+i m \omega}\right) \psi_{1}=-\left(\frac{\frac{1}{K}+M^{2}+i \omega}{1+i m \omega}\right)-\frac{A}{1+i m \omega} \frac{\mathrm{d} \psi_{0}}{\mathrm{~d} y}
\end{gathered}
$$


subject to the boundary conditions

$$
\begin{aligned}
& \psi_{0}=0, \psi_{1}=0 \text { as } y=0 \\
& \psi_{0}=1, \psi_{1}=1 \text { as } y \rightarrow \infty
\end{aligned}
$$

Using the boundary conditions the solution of Equation (14) and Equation (15) are given by respectively

$$
\begin{gathered}
\psi_{0}=1-\mathrm{e}^{-(1+c) y} \\
\psi_{1}=1-\frac{A a(1+c) \mathrm{e}^{(-1-c) y}}{(1+c)^{2}-(1+c) a-a b}-\left[1-\frac{A a(1+c)}{(1+c)^{2}-(1+c) a-a b}\right] \mathrm{e}^{\frac{-a-\sqrt{a^{2}+4 a b}}{2} y}
\end{gathered}
$$

where $a=\frac{1}{1+m i \omega}, b=\frac{1}{K}+M^{2}+i \omega, c=\sqrt{1+4\left(\frac{1}{K}+M^{2}\right)}$

Further

$$
\frac{-a-\sqrt{a^{2}+4 a b}}{2}=-\frac{1+h_{r}+m \omega h_{i}}{2\left(1-m^{2} \omega^{2}\right)}+i \frac{m \omega\left(1+h_{r}\right)-h_{i}}{2\left(1-m^{2} \omega^{2}\right)}=H_{r}+i H_{i}
$$

Therefore the velocity field $u$ is given by

$$
u(y, t)=1-\mathrm{e}^{-(1+c) y}+\varepsilon\left(S_{r} \cos \omega t+S_{i} \sin \omega t\right)
$$

where

$$
\begin{gathered}
S_{r}=1-D \mathrm{e}^{-(1+c) y}+\mathrm{e}^{H_{r} y}\left((D-1) \cos H_{i} y+E \sin H_{i} y\right) \\
S_{i}=\mathrm{e}^{H_{r} y}\left(E \cos H_{i} y-(D-1) \sin H_{i} y\right)-E \mathrm{e}^{-(1+c) y} \\
D=\frac{A(1+c)\left(c(1+c)-\left(\frac{1}{K}+M^{2}\right)\right)}{\left\{(1+c)^{2}-(1+c)-\left(\frac{1}{K}+M^{2}\right)\right\}^{2}} \\
E=\frac{A(1+c)\left(m \omega(1+c)^{2}-\omega\right)}{\left\{(1+c)^{2}-(1+c)-\left(\frac{1}{K}+M^{2}\right)\right\}^{2}}
\end{gathered}
$$

The non-dimensional form of the skin friction at the plate is given by

$$
\tau_{\mid y=0}=(1+c)+\varepsilon|B| \cos (\omega t+\theta)
$$

where

$$
\begin{gathered}
|B|=\sqrt{\left\{D(1+c)+H_{r}(D-1)+E H_{i}\right\}^{2}+\left\{E H_{i}+(D-1) H_{i}+E(1+c)\right\}^{2}} \\
\tan \theta=\frac{E H_{i}+(D-1) H_{i}+E(1+c)}{D(1+c)+H_{r}(D-1)+E H_{i}}
\end{gathered}
$$

\section{Results and Discussions}

In the present study, the fluctuating flow of an unsteady incompressible fluid of second grade type through a porous medium occupying a semi-infinite region of the space bounded by a porous plate has been discussed. The analytical solution for the velocity field has been obtained by the method of Lighthill. The skin friction is also obtained at the plate $(y=0)$. The effects of magnetic field parameter $M$, permeability of porous medium $K$ 
on the fluctuating parts $\left(S_{r}, S_{i}\right)$ of the velocity field have been illustrated graphically. The effect of the frequency of fluctuation $\omega$ on the amplitude of fluctuation $|B|$ of the skin friction has also been illustrated graphically.

Graphical representations have been illustrated to see the effects of Hartmann number $M$, permeability parameter $K$ and normal stress moduli $\alpha$ on the fluctuating parts $\left(S_{r}, S_{i}\right)$ of the velocity field $u$ in the boundary layer. Figure 1 depicts $S_{r}$ against $y$ for different values of Hartmann number $M$. It is clear that increase in $y$ leads to sharp increase in $S_{r}$ in the boundary layer. Furthermore, as $M$ takes higher values $S_{r}$ increases near the plate and it can be noticed that $S_{r}$ asymptotically approaches $U(t)$ as it goes far away from the plate. All the parameters take arbitrary values. Thus the Figure 1 shows the effect of the parameter $M$ on $S_{r}$. The parameters $\varepsilon$ and $A$ are restricted to take values so that $\varepsilon \ll 1$ and $A \varepsilon \leq 1$. In Figure $2, S_{r}$ is depicted against $y$ for different values of permeability parameter $K$. The figure shows that an increase in $y$ leads to sharp increase in $S_{r}$. It is evident from the figure that increase in $K$ leads to decrease in $S_{r}$ i.e. porosity of the medium produces a resistance force in velocity field and furthermore $S_{r}$ asymptotically approaches $U(t)$ as it goes far away from the plate. Thus the figure shows the effect of the permeability parameter $K$ on $S_{r}$ graphically. The parameters $\varepsilon$ and $A$ are restricted to take values so that $\varepsilon \ll 1$ and $A \varepsilon \leq 1$. In Figure $3, S_{r}$ is depicted against $y$ for different values of $K$ with $\alpha=0.0, M=0.0\left(i . e . B_{0}=0.0\right)$ that is the case for graphical illustration made by Varshney in his work. Curve with $M=0$ corresponds to non-MHD flow and $\alpha=0.0$ corresponds to viscoelastic fluid flow other than non-Newtonian. Figure shows that as $K$ takes higher values $S_{r}$ decreases i.e. porosity produces a resistance on the flow and $S_{r}$ approaches $U(t)$ as it goes far away from the plate. The fluctuating part $\left|S_{i}\right|$ is depicted against y for different values of suction parameter $A$ in Figure 4. The figure shows that $\left|S_{i}\right|$ increases and decreases afterwards sharply with $y$ near the boundary layer. It is evident from the figure that as suction parameter $A$ takes higher values $\left|S_{i}\right|$ increases. In Figure $5,\left|S_{i}\right|$ is depicted against $y$ for different values of permeability parameter $K$ with $M=0.0, \alpha=0.0$. It can be seen from the figure that $\left|S_{i}\right|$ increases and decreases afterwards sharply with $y$ near the boundary layer. It shows that as $K$ takes increasing values $\left|S_{i}\right|$ decreases and the curves with $M=0.0, \alpha=0.0$ are related to the flow of fluids other than non-Newtonian in absence of magnetic field $B_{0}$. The fluctuating part $\left|S_{i}\right|$ of the velocity field is depicted against $y$ for different values of magnetic field parameter $M$ and suction parameter $A$ in Figure 6. It is seen from the figure that near the boundary layer the curves go up sharply and then go down rapidly for different values of parameters $M$ and $A$. As $M$ and $A$ increase the picardness of the curves increase near the boundary layer. The velocity curve with $A=0$ corresponds to the flow without suction. In Figure 7, the amplitude $|B|$ through

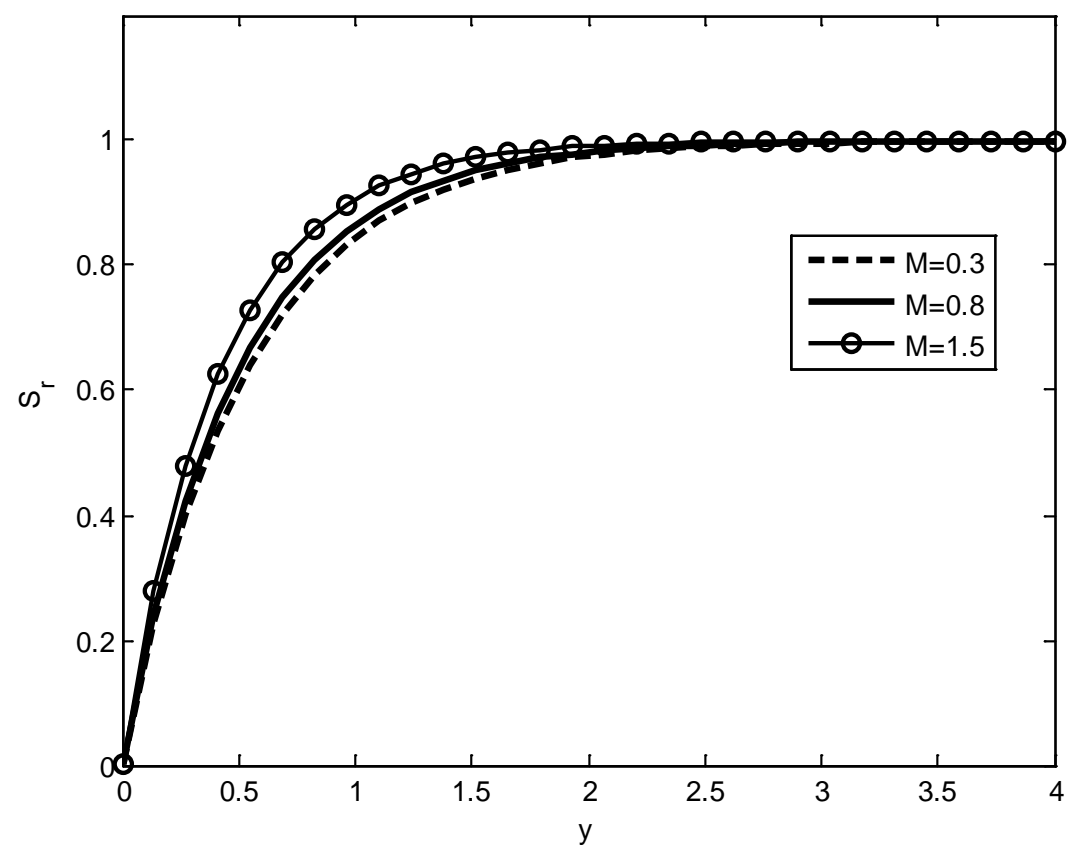

Figure 1. The velocity is depicted against $y$ for different values of magnetic field parameter $M . \quad t=0.1, \omega=0.4, \varepsilon=0.002, K=0.3, m=0.3, A=10$. 


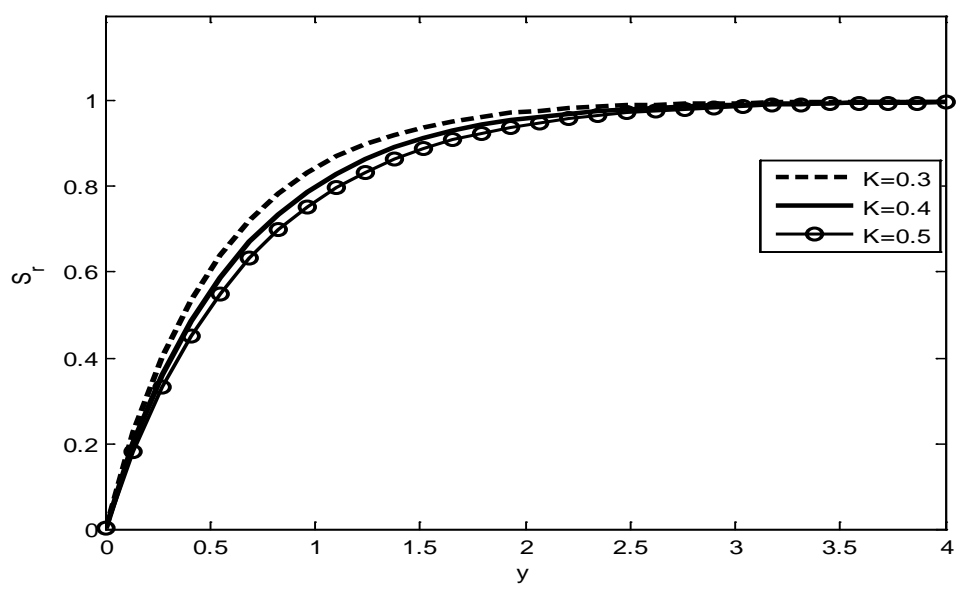

Figure 2. The velocity is depicted against y for different values of permeability parameter K. $t=0.1, \omega=0.4, \varepsilon=0.002, M=0.3, m=0.3, A=10$.

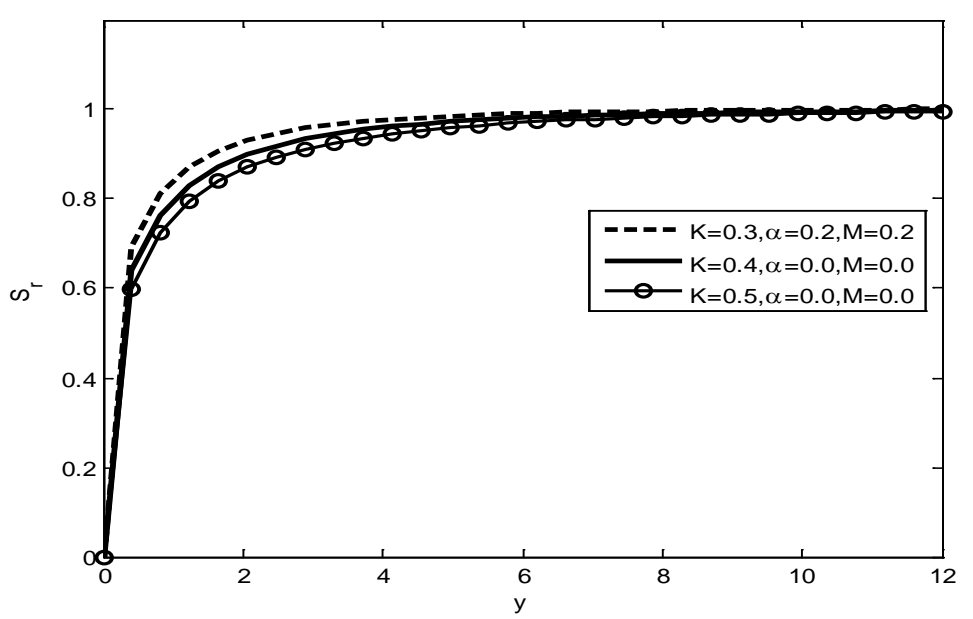

Figure 3. The fluctuating part $S_{r}$ of velocity field is depicted against $y$ for different values of $K, \alpha$ and $M . \quad \varepsilon=0.002, \mu=0.2, \omega=0.4, t=0.1, A=10$.

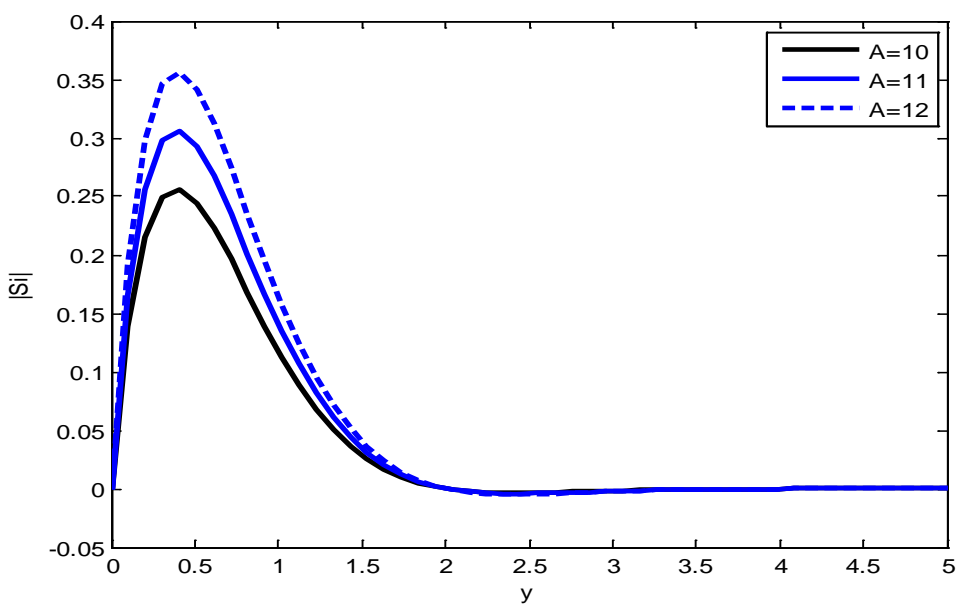

Figure 4. Fluctuating part $\left|S_{i}\right|$ of velocity profile is depicted against y for different values of suction parameter $A$ with $K=0.1, M=0.2, \omega=0.2, \varepsilon=0.002, \alpha=0.1, t=0.2$. 


$$
\frac{N}{n ! n}
$$


which the skin friction fluctuates at the plate is plotted against $\omega$ for different values of permeability parameter $K$ and suction parameter $A$. It is seen from the figure that $|B|$ decreases as the frequency of fluctuation $\omega$ increases. As $K$ as well as $A$ take higher values $|B|$ decreases. In the figure, the curve related to $A=0$ corresponds to the fluid flow without any suction.

\section{References}

[1] Soundalgekar, V.M. and Puri, P. (1969) On Fluctuating Flow of an Elastic-Viscous Fluid past an Infinite Plate with Variable Suction. Journal of Fluid Mechanics, 35, 561-573. http://dx.doi.org/10.1017/S0022112069001297

[2] Shen, F., Tan, W., Zhao, Y. and Masuoka, T. (2006) The Rayleigh-Stokes Problem for a Heated Generalized Second Grade Fluid with Fractional Derivative Model. Nonlinear Analysis: Real World Applications, 7, 1072-1080. http://dx.doi.org/10.1016/j.nonrwa.2005.09.007

[3] Varshney, C.L. (1979) Fluctuating Flow of Viscous Fluid through a Porous Medium Bounded by a Porous Plate. Indian Journal of Pure and Applied Mathematics, 10, 1558-1564.

[4] Lighthill, M.J. (1954) The Response of Laminar Skin Friction and Heat Transfer to Fluctuations in the Stream Velocity. Proceedings of the Royal Society A, 224, 1-23. http://dx.doi.org/10.1098/rspa.1954.0137

[5] Messiha, S.A.S. (1966) Laminar Boundary Layer in Oscillating Flow along an Infinite Flat Plate with Variable Suction. Proceedings of the Cambridge Philosophical Society, 62, 329-337. http://dx.doi.org/10.1017/S030500410003989X

[6] Stuart, J.T. (1955) A Solution of the Navier-Stokes and Energy Equations Illustrating the Response of Skin Friction and Temperature of an Infinite Plate Thermometer to Fluctuations in the Stream Velocity. Proceedings of the Royal Society A, 231, 116-130. http://dx.doi.org/10.1098/rspa.1955.0160

[7] Gholizadeh, A. (1990) MHD Oscillatory Flow past a Vertical Porous Plate through Porous Medium in the Presence of Thermal and Mass Diffusion with Constant Heat Source. Astrophysics and Space Science, 174, 303-310. http://dx.doi.org/10.1007/BF00642515

[8] Moniem, A.A. and Hassanin, W.S. (2013) Solution of MHD Flow past a Vertical Porous Plate through a Porous Medium under Oscillatory Suction. Applied Mathematics, 4, 694-702. http://dx.doi.org/10.4236/am.2013.44096

[9] Venkateswarlu, M., Ramana Reddy, G.V. and Lakshmi, D.V. (2013) Unsteady MHD Flow of a Viscous Fluid past a Vertical Porous Plate under Oscillatory Suction Velocity. Advances in Applied Science Research, 4, 52-67.

[10] Soundalgekar, V.M. and Takhar, H.S. (1977) MHD Oscillatory Flow past a Semi Infinite Plate. AIAA Journal, 15, 457-458. http://dx.doi.org/10.2514/3.60646 